\title{
E-nose herbs recognition system based on artificial neural network technique
}

\begin{abstract}
Electronic sensing technology intervention was intended to overcome human's physical limitation. It has developed and greatly advanced over the decade. This project emphasizes on characterizing herbs species based on unique of herbs odor. E-nose system in this project consist an array of commercial gas sensor which detects gas through an increase in electrical conductivity when reducing gases are absorbed on the sensor's surface. Data obtained from sensors array are classified using Artificial Neural Network (ANN) technique. The E-nose system with five sensors has the highest capability in classifying herbs sample. Accuracy in classifying the correct herbs increases with number of the sensors used. Results show that sensitivity of E-nose towards herbs classification increases with higher number of sensors.
\end{abstract}

Keyword: Electronic nose; Artificial neural network; Array of sensor; Classification 